\title{
gs \\ Measurement of ultralow vertical emittance using a calibrated vertical undulator
}

\author{
K. P. Wootton, ${ }^{1, *}$ M. J. Boland, ${ }^{1,2}$ and R. P. Rassool ${ }^{1}$ \\ ${ }^{1}$ School of Physics, The University of Melbourne, Parkville, VIC 3010, Australia \\ ${ }^{2}$ Australian Synchrotron, 800 Blackburn Rd, Clayton, VIC 3168, Australia
}

(Received 7 July 2014; published 14 November 2014)

\begin{abstract}
Very few experimental techniques are useful for the direct observation of ultralow vertical emittance in electron storage rings. In this work, quantitative measurements of ultralow (pm rad) electron beam vertical emittance using a vertical undulator are presented. An undulator radiation model was developed using the measured magnetic field of the APPLE-II type undulator. Using calibrated experimental apparatus, a geometric vertical emittance of $\varepsilon_{y}=0.9 \pm 0.3 \mathrm{pm}$ rad has been observed. These measurements could also inform modeling of the angular distribution of undulator radiation at high harmonics, for proposed diffraction-limited storage ring light sources.
\end{abstract}

DOI: 10.1103/PhysRevSTAB.17.112802

PACS numbers: 29.27.-a, 41.75.Ht, 41.60.-m, 41.85.Lc

\section{INTRODUCTION}

The aim of the present work was to calibrate a vertical undulator for the direct quantitative measurement of vertical emittance of an electron beam in a storage ring. The present study is significant because it is the first quantitative measurement of the electron beam vertical emittance in a storage ring using radiation from a vertical undulator.

Previous work using simulations demonstrated that radiation from vertical undulators was sensitive to vertical beam emittances [1]. Vertical undulators are so defined because the electron beam is deflected perpendicular to the (usually horizontal) orbit plane of the storage ring. Recent experiments have demonstrated that radiation from vertical undulators is sensitive to ultralow electron beam vertical emittances [2].

At present, very few measurement techniques are useful for the direct observation of ultralow ( $\mathrm{pm}$ rad) vertical emittance in electron storage rings [3]. This emittance range is of contemporary importance for proposed electronpositron collider damping rings, to optimize for maximum luminosity $[4,5]$. These vertical emittances will be achieved using low-emittance tuning optimization routines, which are the subject of active research at many electron storage ring light sources [6-12]. The effective use and experimental verification of these optimization routines rely upon measurement of vertical emittance.

This paper addresses the calibration of a vertical undulator for the direct measurement of vertical emittance. The paper is organized as follows. The theory of spontaneous

\footnotetext{
*k.wootton@student.unimelb.edu.au

Published by the American Physical Society under the terms of the Creative Commons Attribution 3.0 License. Further distribution of this work must maintain attribution to the author(s) and the published article's title, journal citation, and DOI.
}

undulator radiation is summarized in order to motivate the choice of experimental apparatus. Measurements of the APPLE-II undulator radiation are presented, with particular emphasis given to ultralow vertical emittances. Finally, implications of this measurement technique are outlined, with particular reference to proposed diffraction-limited storage ring (DLSR) light sources.

\section{UNDULATOR THEORY}

The theory underpinning this emittance measurement is the convolution of the electron beam distribution with the single electron angular distribution of spontaneous radiation from a planar undulator [13]. For an undulator of ideal sinusoidal field distribution, the spectral and angular distribution of flux from a single electron filament is given in terms of linear polarizations $\sigma$ and $\pi$ at the photon frequency $\omega$ as a sum over all undulator harmonics $n$ by [14]

$$
\begin{aligned}
\frac{\mathrm{d} N_{\mathrm{ph}}(\omega)}{\mathrm{d} \Omega}= & \alpha \gamma^{2} N^{2} \frac{\Delta \omega}{\omega} \frac{I}{q_{e}} \\
& \times \sum_{n=1}^{\infty} n^{2}\left[\frac{\sin \varphi(n)}{\varphi(n)}\right]^{2}\left[D_{\sigma}(n)^{2}+D_{\pi}(n)^{2}\right],
\end{aligned}
$$

where

$$
\begin{gathered}
\varphi(n)=\pi N \frac{\Delta \omega_{n}}{\omega_{1}}, \\
D_{\sigma}(n)=\frac{2 \gamma \theta \Sigma_{1}(n) \cos \phi-K \Sigma_{2}(n)}{1+\frac{1}{2} K^{2}+\gamma^{2} \theta^{2}}, \\
D_{\pi}(n)=\frac{2 \gamma \theta \Sigma_{1}(n) \sin \phi}{1+\frac{1}{2} K^{2}+\gamma^{2} \theta^{2}}
\end{gathered}
$$


(a) $D_{\sigma}(1)$

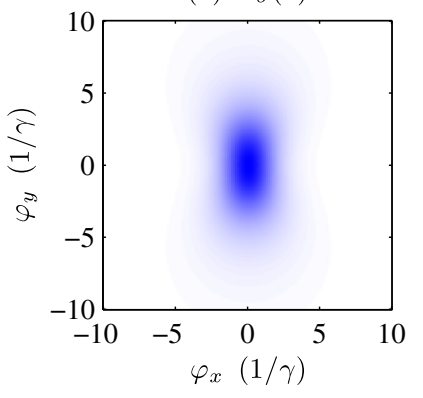

(c) $D_{\sigma}(14)$

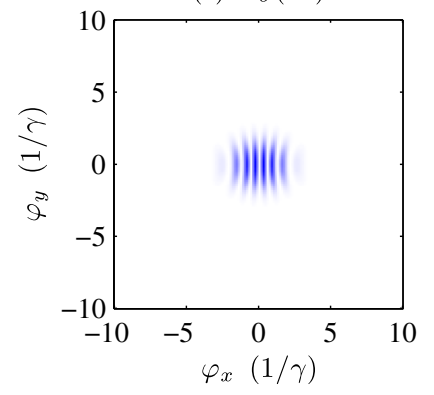

(e) $D_{\sigma}(15)$

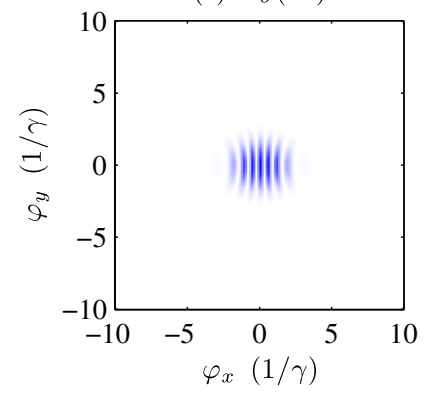

(b) $D_{\pi}(1)$

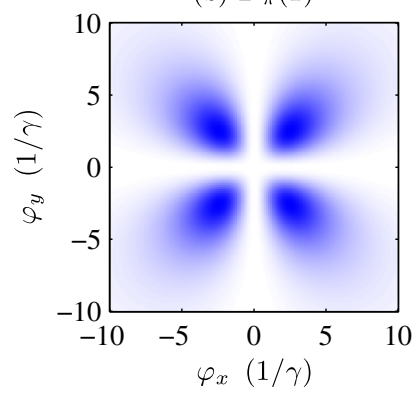

(d) $D_{\pi}(14)$

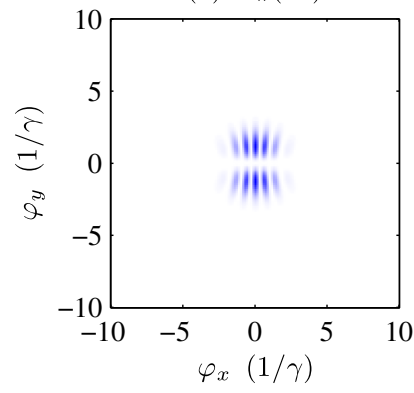

(f) $D_{\pi}(15)$

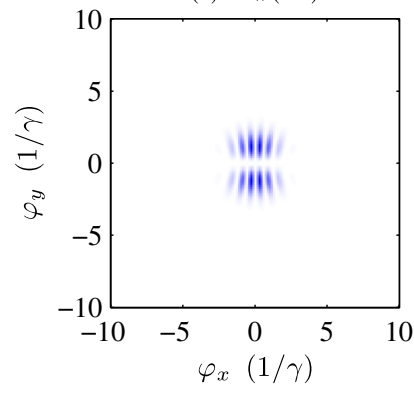

FIG. 1. Analytical single-electron angular distribution of undulator radiation for an electron in a horizontal, planar undulator. Both $D_{\sigma}(n)$ and $D_{\pi}(n)$ linear polarization modes [Eq. (3), (4)] are illustrated for undulator harmonics $n=1,14,15$ [14]. Electron and undulator parameters are summarized in Table I. The angular distribution is plotted in terms of horizontal and vertical opening angles $\varphi_{x}, \varphi_{y}$, in units of the angle $1 / \gamma$. For high undulator harmonics, the distribution resembles a narrow interference pattern.

and $\alpha$ is the fine-structure constant, $\gamma$ is the Lorentz factor of beam electrons, $N$ is the number of undulator periods, $I$ the stored electron beam current, $q_{e}$ the electronic charge, $K$ the undulator deflection parameter, $\omega_{n}$ the photon frequency of the $n$th undulator harmonic $\left(\omega_{1}\right.$ for the fundamental), and $\Delta \omega_{n}=\omega-\omega_{n}$. The distributions are

TABLE I. Electron and undulator parameters for Fig. 1.

\begin{tabular}{lcc}
\hline \hline Parameter & Symbol & Value \\
\hline Lorentz factor & $\gamma$ & 5870 \\
Undulator periods & $N$ & 25 \\
Deflection parameter & $K$ & 5 \\
\hline \hline
\end{tabular}

expressed in the polar angle $\theta$ and azimuthal angle $\phi$. The sums $\Sigma_{1}(n)$ and $\Sigma_{2}(n)$ are given by

$$
\begin{gathered}
\Sigma_{1}(n)=\sum_{m=-\infty}^{\infty} J_{-m}(u) J_{n-2 m}(v), \\
\Sigma_{2}(n)=\sum_{m=-\infty}^{\infty} J_{-m}(u)\left[J_{n-2 m-1}(v)+J_{n-2 m+1}(v)\right],
\end{gathered}
$$

where $J$ are Bessel functions of the first-kind, with arguments of

$$
\begin{gathered}
u=\frac{\omega}{\omega_{1}} \frac{\beta K^{2}}{4\left(1+\frac{1}{2} K^{2}+\gamma^{2} \theta^{2}\right)}, \\
v=\frac{\omega}{\omega_{1}} \frac{2 \beta K \gamma \theta \cos \phi}{1+\frac{1}{2} K^{2}+\gamma^{2} \theta^{2}},
\end{gathered}
$$

where $\beta$ is the ratio of the velocity of the electron to the speed of light $(\beta=v / c \approx 1)$.

As an example, this single-electron angular distribution of radiation for a usual horizontal undulator is illustrated in Fig. 1, for both the $\sigma$ and $\pi$ polarizations of individual undulator harmonics [Eqs. (3), (4)]. The electron and undulator parameters used in these calculations are summarized in Table I.

Figure 1 highlights that for the first undulator harmonic $n=1$ [Fig. 1(a)], the angular distribution of undulator radiation could be appropriately approximated by a Gaussian distribution. However it is noted in Ref. [15] that this Gaussian approximation has not explicitly been demonstrated. This approximation breaks down at unusually high deflection parameters and high harmonics. For undulator harmonics $n=14,15$ the single-electron angular distribution of undulator radiation resembles an interference pattern [Fig. 1(c,e)]. This pattern is significantly narrower than the usual $1 / \gamma$ cone of undulator radiation at the first harmonic. The convolution of this singleelectron distribution with the electron beam is a probe which is sensitive to electron beam emittance. Hence, a vertical undulator is used specifically for sensitivity to vertical electron beam emittance.

\section{EXPERIMENTAL METHOD}

The technique presented in this work employs two main ideas: a vertical undulator as a photon source [1], and the measurement of ratios of photon beam brilliance for adjacent undulator harmonics rather than fitting absolute values [2].

\section{A. Undulator}

Vertical insertion devices are seldom used in electron storage ring light sources, and are selected only when there is a compelling technical need for an insertion device to 
TABLE II. Electron beam and undulator parameters for this experiment and simulation [20].

\begin{tabular}{lccc}
\hline \hline Parameter & Symbol & Value & Units \\
\hline Electron beam & & & \\
Energy & $E$ & 3.033 & $\mathrm{GeV}$ \\
Energy spread & $\sigma_{E} / E$ & 0.0011 & $\ldots$ \\
Horizontal emittance & $\varepsilon_{x}$ & 10 & $\mathrm{~nm} \mathrm{rad}$ \\
Undulator & $K$ & $3.84^{\mathrm{a}}$ & \\
Deflection parameter & $\lambda_{u}$ & 75 & $\ldots$ \\
Period length & $N$ & 25 & $\ldots$ \\
Number of full periods & $N$ \\
\hline \hline
\end{tabular}

${ }^{\mathrm{a}}$ Fitted value. The measured undulator magnetic field map was used in simulations rather than an ideal sinusoidal field distribution [20].

adopt such an orientation [16]. For reasons of maintaining a large momentum aperture for beam injection, typically beams are deflected in the orbit (horizontal) plane [16,17].

There are no vertical undulators at the $3 \mathrm{GeV}$ Australian Synchrotron (AS) storage ring. In this work, an ellipticallypolarizing Advanced Planar Polarized Light Emitter (APPLE-II) undulator was phased as a vertical undulator $[18,19]$. The undulator was closed to a gap of $17.1 \mathrm{~mm}$ (close to the minimum gap), and moved to a row phase of $37.5 \mathrm{~mm}$, corresponding to half an undulator period. Insertion device and beam parameters for this experiment are summarized in Table II.

\section{B. Undulator simulations}

A numerical model of radiation from this APPLE-II insertion device was developed. The magnetic field of the APPLE-II insertion device used in this experiment (Sec. III A) was previously measured using a three-axis Hall probe magnetometer, at a magnetic gap of $16 \mathrm{~mm}$ [19]. This gap results in stronger fields (higher undulator $K$ ) than the present experiments at a gap of $17.1 \mathrm{~mm}$. Hence, the magnitude of the measured magnetic field was scaled down to simulate this experiment. Using this scaled magnetic field, the spectral and spatial distribution of undulator radiation was simulated using the synchrotron radiation simulation code SPECTRA [21]. Further details on modeling are given in Ref. [20].

\section{Storage ring and electron beam}

To optimize the AS electron storage ring for ultralow vertical emittance, orbit response matrices were measured with the skew quadrupole magnets unpowered [22]. Using the orbit response matrices fitted by LOCO [23], the strengths of skew quadrupole magnets were optimized for a range of vertical emittances, including the minimum emittance fitted by the optimization routine [24]. This model was used to define vertical emittance setpoints $\epsilon_{y}$.

\section{Photon beam line}

A soft X-ray photon beam line was used to measure the undulator radiation. This apparatus is illustrated schematically in Fig. 2 [25]. The apparatus is very similar to that of other recent experiments with APPLE-II undulators in electron storage rings [26].

Notably, the beam line used in the present experiment does not have a fixed-size pinhole for characterizing the spatial profile of undulator radiation. Hence, four straight blades of the white beam slits were closed from above, below, left and right of the photon beam to form a pinhole aperture. In previous experiments, it was not possible to simultaneously minimize the position and height of the pinhole. As a consequence, instead of measuring the electron beam size, the dimension of the pinhole aperture was fitted [2,27]. In the present work, the pinhole was made as small as possible, while still passing photons. Repeated measurements of the photon flux while closing the aperture are illustrated in Fig. 3. Using this, a pinhole height of $5 \pm 5 \mu \mathrm{m}$ was set.

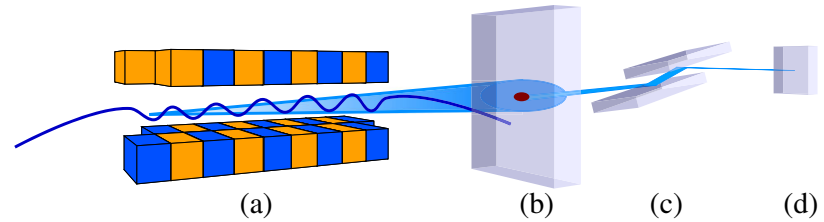

FIG. 2. Schematic of vertical undulator emittance measurement apparatus. This soft X-ray photon beam line is further described in Ref. [25]. (a) APPLE-II undulator in storage ring; (b) Pinhole aperture formed to spatially filter undulator radiation; (c) Grating monochromator to bandpass photon energy; (d) Silicon photodiode to measure photon beam intensity.

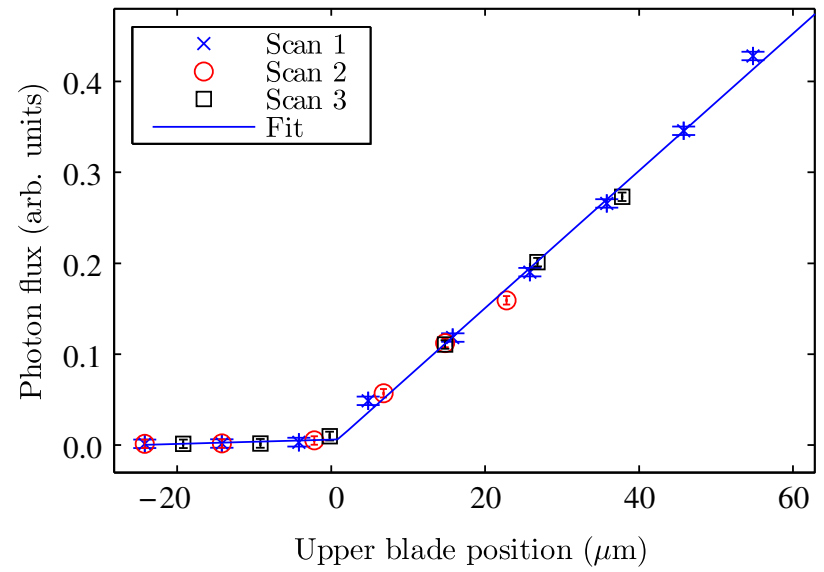

FIG. 3. Measured photon flux passing white-beam slits, at a photon energy of $2015 \mathrm{eV}$, for different positions of the upper blade. The pinhole aperture was measured by closing the upper blade until the photon flux passing the pinhole was minimized. For negative positions of the upper blade, the upper and lower blades are crossed, and (ideally) no photon flux passes. A pinhole height of $5 \pm 5 \mu \mathrm{m}$ was selected. 


\section{E. Orbit bumps}

In the present experiment, the need to center the pinhole with the central axis of undulator radiation was obviated by the decision to bump the electron beam orbit through the undulator. This is illustrated schematically in Fig. 4.

Closed orbit bumps using six vertical correctors were calculated using the AT [28] and MIDDLE LAYER [29] codes. The electron beam vertical angle through the undulator $(v \equiv \mathrm{d} y / \mathrm{d} s)$, arising from these bumps, was measured using storage ring electron beam position monitors adjacent to the undulator. The angular profile of undulator radiation using vertical orbit bumps is illustrated for undulator harmonics $n=13,14$, and 15 in Fig. 5. From these measurements, the vertical center of the distribution of undulator radiation corresponded to an angular bump of $v=6 \mu \mathrm{rad}$ from the nominal beam orbit.

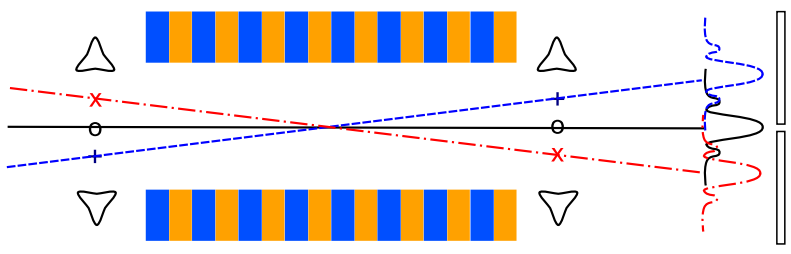

FIG. 4. Schematic of vertical electron beam orbit bumps through an insertion device, at a single photon energy corresponding to an odd undulator harmonic. From an initial orbit through vertical positions denoted by $\circ$, orbit correctors change the electron beam trajectory angle $v$ measured at beam position monitors to $x$ and + . This varies the angle of insertion device radiation measured passing the fixed pinhole aperture.

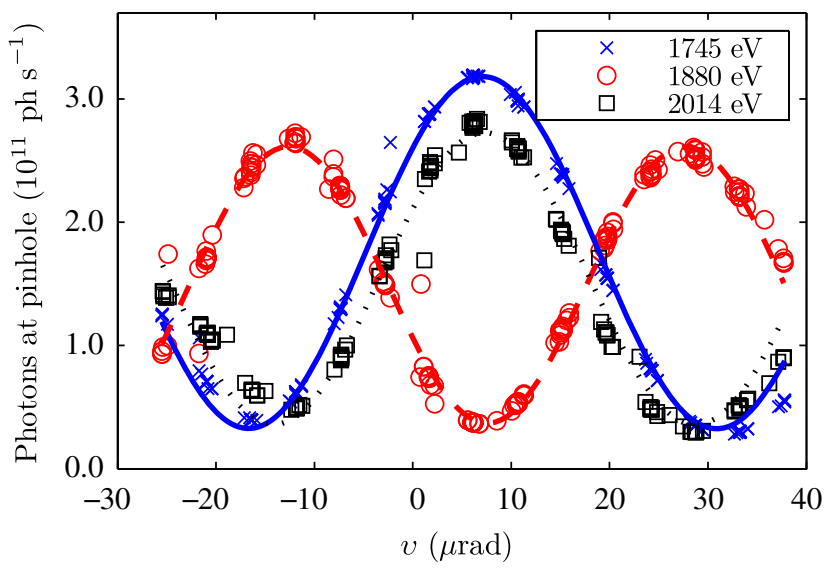

FIG. 5. Measured pinhole photon flux, fitted for orbit bumps through the undulator. Storage ring skew quadrupoles were configured for minimum vertical emittance $\left(\varepsilon_{y}<1 \mathrm{pm} \mathrm{rad}\right)$. Photon energies correspond to undulator harmonics $n=13$, 14 , and 15 [30].

\section{RESULTS}

With the vertical center of the undulator radiation distribution defined at an angle of $v=6 \mu \mathrm{rad}$, angular bumps were made within $5 \mu \mathrm{rad}$ of this center angle. This measured profile is illustrated in Fig. 6 for undulator harmonics $n=13,14$, and 15 .

The flux ratio of the 14th to 15th harmonics of undulator radiation passing the pinhole $\left(F_{14} / F_{15}\right)$ was measured using orbit bumps [2]. These harmonics were selected as the highest harmonics (greatest sensitivity to vertical emittance) which were still lower in photon energy than the $\mathrm{Au}$ absorption edge cutoff of $2150 \mathrm{eV}$ of the beam line mirrors [25]. For different model values of vertical emittance, the flux ratio measured at the angle of symmetry in the radiation distribution $v=6 \mu \mathrm{rad}$ is presented in Fig. 7 . Also illustrated are simulations of the flux ratio using the

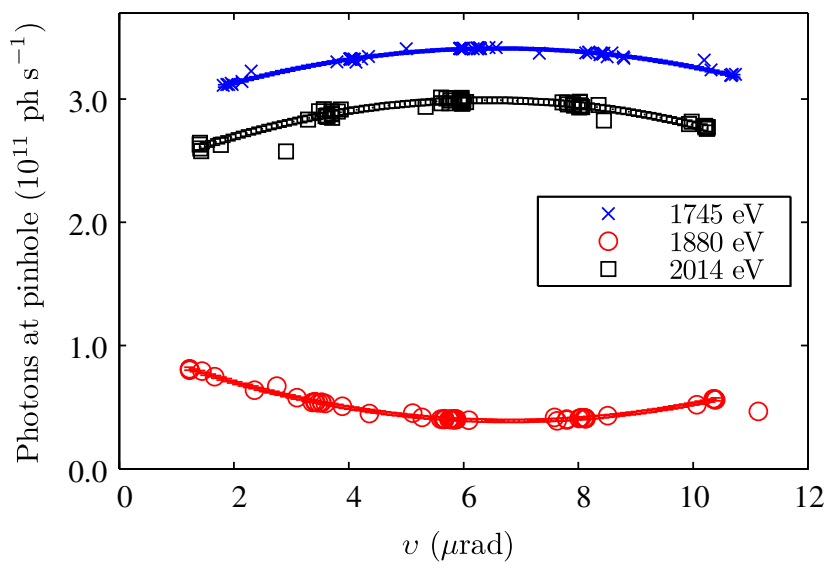

FIG. 6. Measured pinhole photon flux fitted for orbit bumps through the undulator. Measured data at harmonics $n=13,14$, and 15 are fitted by parabolas, with the $1 \sigma$ statistical distribution of measured data points illustrated [30].

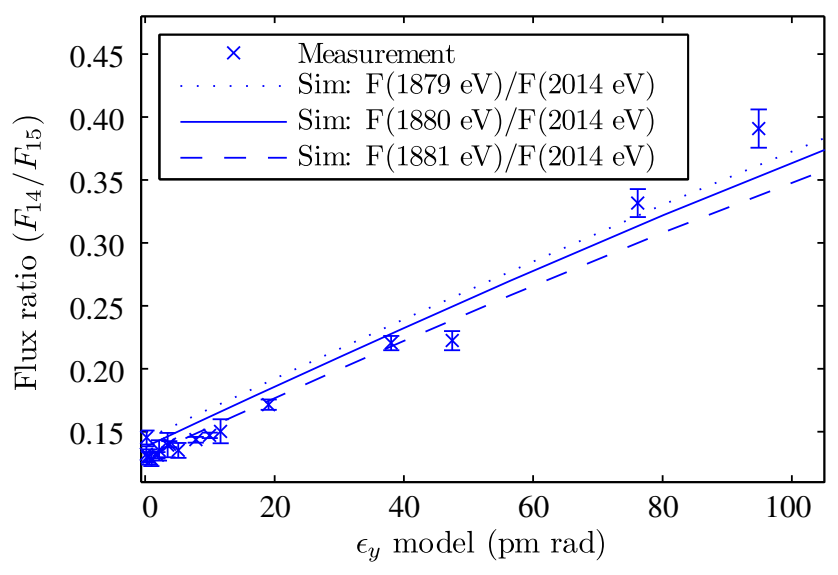

FIG. 7. Measurement of undulator radiation flux ratios using orbit bumps through a vertical undulator at the AS storage ring, for various vertical emittances $\epsilon_{y}$. Simulations are plotted with uncertainty in the photon energy scale. 


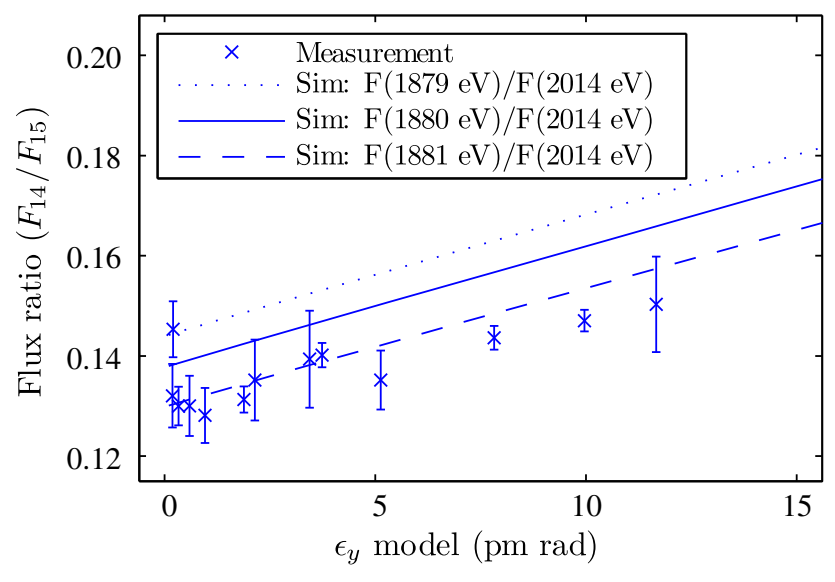

FIG. 8. Magnification of Fig. 7 for small vertical emittances.

model of Sec. III B, evaluated for $F_{14} / F_{15} \equiv$ $F(1880 \mathrm{eV}) / F(2014 \mathrm{eV})$, and with uncertainty in the selection of photon energy of the 14th harmonic of $1880 \pm 1 \mathrm{eV}$. Figure 8 shows a magnification of the measurements of small vertical emittance.

\section{ANALYSIS}

Many physical processes serve to increase the vertical emittance of a beam in an electron storage ring. Singleparticle sources of vertical emittance relevant to this measurement technique were considered.

In particular, this measurement relies on vertical orbit bumps through the insertion device, and adjacent sector multipoles. In an Appendix, it is shown that the singleparticle vertical emittance increase $\Delta \varepsilon_{y}(\nu)$, resulting from these vertical orbit bumps of angle $\nu$, is negligible.

The quantum limit of vertical emittance represents the lower limit of the equilibrium vertical emittance of electron beams. It is governed by the stochastic emission of synchrotron radiation photons in a narrow cone of opening angle $\sim 1 / \gamma$, giving rise to a stochastic distribution of vertical momenta of beam electrons. The quantum limit of vertical emittance is given by $[31,32]$

$$
\varepsilon_{y \mathrm{QLOVE}}=\frac{13}{55} \frac{C_{q}}{\mathcal{J}_{y}} \frac{\oint \beta_{y}(s)\left|G^{3}(s)\right| d s}{\oint\left|G^{2}(s)\right| d s},
$$

with

$$
C_{q}=\frac{55 \hbar}{32 \sqrt{3} m c},
$$

where $\mathcal{J}_{y}$ is the vertical damping partition number, $\beta_{y}(s)$ the vertical betatron function and $G \equiv 1 / \rho(s)$, where $\rho(s)$ is the local bending radius. As noted by Sands, the equilibrium vertical quantum limit is independent of the beam energy $\gamma$, and approximately a factor of $1 / \gamma^{2}$ smaller than the equilibrium horizontal emittance [31]. For parameters of the AS storage ring lattice [33], this represents a contribution of $\varepsilon_{y \mathrm{QLOVE}}=0.35 \mathrm{pm} \mathrm{rad}$.

For several model values of vertical emittance, the vertical emittance was measured using both a vertical undulator $\left(\varepsilon_{y \mathrm{VU}}\right)$ and orbit response matrices $\left(\varepsilon_{y \mathrm{ORM}}\right)$. The vertical emittance measured using this vertical undulator technique was calculated using simulations of flux ratios for $F(1881 \mathrm{eV}) / F(2014 \mathrm{eV})$ outlined in Sec. IV. The vertical emittance $\varepsilon_{y \text { ORM }}$ was evaluated using the matrix method of Chao [34], for lattices fitted by LOCO to measured orbit response matrices [24], with uncertainty expressing the standard deviation of vertical emittance fitted around the ring. Using this method, orbit response matrix measurements account only for betatron coupling and vertical dispersion contributions to the vertical emittance, which are normally the dominant contributions to vertical emittance. At these ultralow vertical emittances, for comparison with measurements using a vertical undulator $\left(\varepsilon_{y \mathrm{VU}}\right)$, the vertical emittance measured using orbit response matrices was added in quadrature with the quantum limit of vertical emittance, $\varepsilon_{y \mathrm{OQ}}=\sqrt{\varepsilon_{y \mathrm{ORM}}{ }^{2}+\varepsilon_{y \mathrm{QLOVE}}{ }^{2}}$. These results are illustrated in Fig. 9.

Figure 9 shows that as expected, the contribution of the quantum limit to $\varepsilon_{y \mathrm{OQ}}$ is significant only for vertical emittances less than $1 \mathrm{pm}$ rad. The measured emittances $\varepsilon_{y \mathrm{VU}}$ and $\varepsilon_{y \mathrm{OQ}}$ are in agreement, within the uncertainties of the techniques. The exception is the smallest modeled vertical emittance of $\epsilon_{y}=0.2 \mathrm{pmrad}$, where vertical

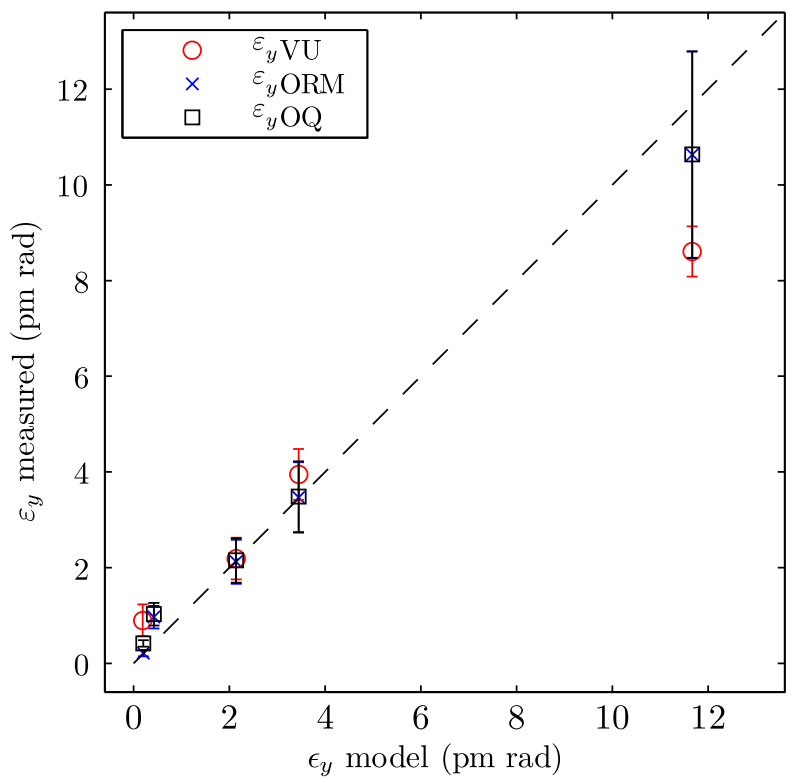

FIG. 9. Measurement of vertical emittance, using orbit bumps through vertical undulator $\left(\varepsilon_{y \mathrm{VU}}\right)$ and orbit response matrix fitting with LOCO [23] ( $\left.\varepsilon_{\text {yORM }}\right)$. The vertical emittance measured using orbit response matrices is also shown in quadrature $\left(\varepsilon_{y \mathrm{OQ}}\right)$ with the contribution from the quantum limit. A dashed line - - is used to indicate measured vertical emittance equal to the model. 
emittances measured are $\varepsilon_{y \mathrm{ORM}}=(0.2 \pm 0.1) \mathrm{pm} \mathrm{rad}$, $\varepsilon_{y \mathrm{OQ}}=(0.4 \pm 0.1) \mathrm{pmrad}$ and $\varepsilon_{y \mathrm{VU}}=(0.9 \pm 0.3) \mathrm{pm} \mathrm{rad}$. Though $\varepsilon_{y \mathrm{OQ}}$ and $\varepsilon_{y \mathrm{VU}}$ are not in agreement within experimental uncertainties, this emittance measured using a direct measurement technique is equal in magnitude to the current record low vertical emittance of Ref. [8].

\section{DISCUSSION}

The principal advantage of this orbit bump approach is that it overcomes the optimization problem of Ref. [2], the need for vertical centering of the pinhole. In the present work, by minimizing only the size of the pinhole, it was possible to directly measure ultralow electron beam vertical emittances.

A limitation of this technique is that for high undulator harmonics, the flux ratio approaches a finite value while beam emittance approaches zero. This is the result of measuring the flux from both the horizontal and vertical linear undulator radiation polarizations. In other vertical emittance measurement techniques, the horizontal polarization component can be rejected using visible light, broadband polarizing filters $[35,36]$. At present, no such broadband linear polarization analyzer exists in the soft $\mathrm{X}$-ray region of the electromagnetic spectrum. The availability of one would extend the linearity of the flux ratio to down to approximately $0.5 \mathrm{pm}$ rad vertical emittance $[1,30]$. The DiagOn device is a soft X-ray imaging detector with linear polarization selection at a single fixed photon energy [37], which may be another experimental option for direct measurement of vertical emittance using soft X-ray undulators.

This measurement of undulator radiation from low vertical emittance beams has direct implications for proposed low-emittance storage ring light sources. Low emittance light sources are beginning to produce undulator radiation of interesting spatial distributions [26]. DLSRs are currently proposed with horizontal emittance of order $100 \mathrm{pm}$ rad [38-44]. Proposed low-emittance light sources and beam lines should be aware of the departure from usual Gaussianapproximated, emittance-dominated photon beams.

The present work has considered spontaneous radiation from ultralow vertical emittance electron beams in storage rings. A recent proposal for a storage-ring based freeelectron laser makes use of flat beams $\left(\varepsilon_{y} \ll \varepsilon_{x}\right)$ with ultralow vertical emittance, based on a gradient vertical insertion device $[45,46]$. For such a proposal, it may be beneficial to consider the angular distribution of stimulated undulator radiation at undulator harmonics greater than the fundamental.

\section{CONCLUSION}

Quantitative measurement of ultralow electron beam vertical emittances was achieved by measuring radiation from an electron beam in a vertical undulator. Direct emittance measurements made using the present technique agreed with indirect emittance measurements evaluated from measured orbit response matrices. Using this vertical undulator technique, a minimum vertical emittance of $\varepsilon_{y}=0.9 \pm 0.3 \mathrm{pmrad}$ has been observed, equal to the present low vertical emittance record of Ref. [8].

This is an extended article of measurements presented at recent International Beams Instrumentation Conferences $[20,27,30]$.

\section{ACKNOWLEDGMENTS}

This research was undertaken on the soft X-ray beam line and storage ring at the Australian Synchrotron, Victoria, Australia. K. P. W. gratefully acknowledges useful discussions with Takashi Tanaka (SPring-8) regarding the implementation of measured magnetic field distributions within the SPECTRA code. K. P. W. gratefully acknowledges useful discussions with Rohan Dowd, Yaw-Ren Eugene Tan and Bruce C. C. Cowie (Australian Synchrotron) during these experiments. K. P. W. gratefully acknowledges the David Lachlan Hay Memorial Fund (The University of Melbourne) for financial support during the preparation of this article.

\section{APPENDIX: EMITTANCE INCREASE FROM ORBIT BUMPS THROUGH MULTIPOLES}

This measurement relies on vertical orbit bumps through the insertion device. The locations of vertical orbit correctors in the AS storage ring necessitates orbit bumps through the sector quadrupole and sextupole magnets, with a sixcorrector closed bump of vertical angle $v$ illustrated in Fig. 10.

These closed bumps give rise to skew quadrupole and dipole fields, and hence vertical emittance growth due to betatron coupling and spurious vertical dispersion.

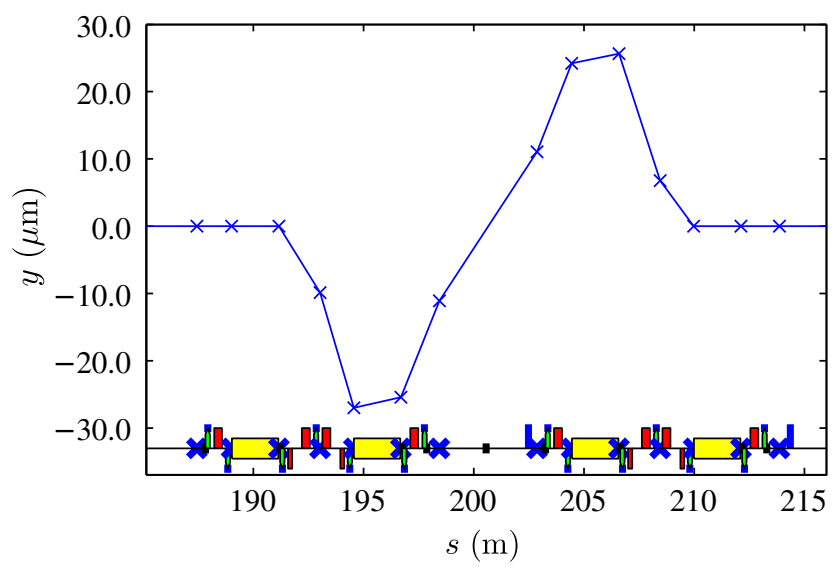

FIG. 10. Simulation of an example orbit bump using six vertical correctors, resulting in angle $v=5 \mu \mathrm{rad}$ within the insertion at the longitudinal position $s=200 \mathrm{~m}$. Longitudinal positions of AS storage ring multipole magnets are indicated [33]. 


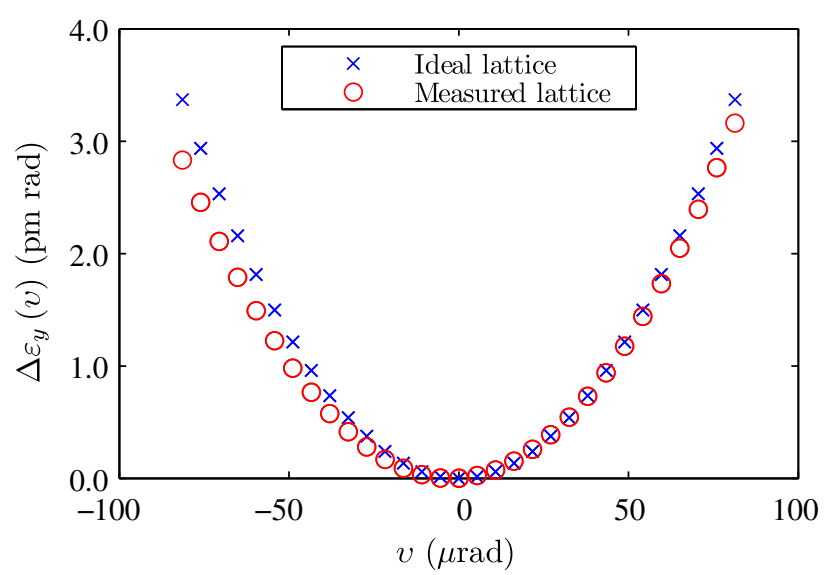

FIG. 11. Simulation of increase in equilibrium vertical emittance $\Delta \varepsilon_{y}(v)=\varepsilon_{y}(v)-\varepsilon_{y}(0)$ for closed angular bumps through one sector of the AS storage ring, for two lattices. The ideal lattice has no magnet misalignments and no skew quadrupole components. The measured lattice includes upright and skew quadrupole errors fitted to a measured orbit response matrix of the AS storage ring.

This vertical emittance increase due to an orbit bump of angle $v$ is denoted by

$$
\Delta \varepsilon_{y}(v)=\varepsilon_{y}(v)-\varepsilon_{y}(0),
$$

where $\varepsilon_{y}(0)$ corresponds to the equilibrium vertical emittance of the lattice with an orbit bump angle of $v=0$ (no bump). Simulations demonstrate that the orbit bumps used in this experiment result in a negligible increase in equilibrium vertical emittance.

Two storage ring lattices were considered in simulation. The equilibrium vertical emittance of an ideal lattice was simulated. The equilibrium vertical emittance resulting from betatron coupling and vertical dispersion for this ideal lattice was $\varepsilon_{y}(0)=0 \mathrm{pm}$ rad. A second lattice was simulated, with lattice elements fitted to a measured orbit response matrix of the AS storage ring. For this lattice, the equilibrium vertical emittance arising from betatron coupling and vertical dispersion is $\varepsilon_{y}(0)=0.2 \mathrm{pm} \mathrm{rad}$.

Evaluating $\Delta \varepsilon_{y}(v)$ permits direct comparison of the vertical emittance arising from the six-corrector orbit bump for both lattices. As illustrated in Fig. 11, both are in agreement.

For the bumps used in these experiments of magnitude $|v|=10 \mu \mathrm{rad}$, the corresponding increase in vertical emittance is not greater than $\Delta \varepsilon_{y}(10)=(0.07 \pm 0.01) \mathrm{pm} \mathrm{rad}$. Increasing the vertical emittance by $\Delta \varepsilon_{y}=1 \mathrm{pm} \mathrm{rad}$ requires an orbit bump of $v=50 \mu \mathrm{rad}$ for this lattice. An orbit bump of this magnitude is not useful for this emittance measurement technique, because it is significantly larger than the angular distribution of the interference pattern illustrated in Fig. 5.
[1] S. Takano, in Proceedings of the Workshop on Precise Measurements of Electron Beam Emittances, KEK Proceedings, Vol. 97-20, edited by J. Urakawa, S. Kamada, K. Nakajima, and T. Mitsuhashi (Tsukuba, Ibaraki, Japan, 1997), p. 20.

[2] K. P. Wootton, M. J. Boland, R. Dowd, Y.-R.E. Tan, B. C.C. Cowie, Y. Papaphilippou, G. N. Taylor, and R. P. Rassool, Phys. Rev. Lett. 109, 194801 (2012).

[3] J. Flanagan, in Proceedings of the 2nd International Particle Accelerator Conference, San Sebastián, Spain (EPS-AG, Spain, 2011), p. WEYB01.

[4] P. Lebrun, L. Linssen, A. Lucaci-Timoce, D. Schulte, F. Simon, S. Stapnes, N. Toge, H. Weerts, and J. Wells, CERN Report No. CERN-2012-005, 2012.

[5] T. Behnke, J. E. Brau, B. Foster, J. Fuster, and M. Harrison, ILC Report No. ILC-REPORT-2013-040, 2013.

[6] A. Franchi, L. Farvacque, J. Chavanne, F. Ewald, B. Nash, K. Scheidt, and R. Tomás, Phys. Rev. ST Accel. Beams 14, 034002 (2011).

[7] S. M. Liuzzo, M. E. Biagini, P. Raimondi, M. Aiba, and M. Böge, in Proceedings of the 3rd International Particle Accelerator Conference, New Orleans, LA, 2012 (IEEE, Piscataway, NJ, 2012), p. TUOAA03.

[8] M. Aiba, M. Böge, N. Milas, and A. Streun, Nucl. Instrum. Methods Phys. Res., Sect. A 694, 133 (2012).

[9] M. Takao, M. Masaki, Y. Shimosaki, K. Soutome, S. Takano, and C. Zhang, in Proceedings of the 3rd International Particle Accelerator Conference, New Orleans, LA, 2012 (IEEE, Piscataway, NJ, 2012), p. TUPPC016.

[10] X. Huang, J. Corbett, J. Safranek, and J. Wu, Nucl. Instrum. Methods Phys. Res., Sect. A 726, 77 (2013).

[11] C. Liu, Y. Luo, and M. Minty, Nucl. Instrum. Methods Phys. Res., Sect. A 750, 56 (2014).

[12] J. Shanks, D. L. Rubin, and D. Sagan, Phys. Rev. ST Accel. Beams 17, 044003 (2014).

[13] K.-J. Kim, Nucl. Instrum. Methods Phys. Res., Sect. A 246, 71 (1986).

[14] H. Wiedemann, Particle Accelerator Physics, 3rd ed. (Springer-Verlag, Berlin, Germany, 2007).

[15] T. Tanaka, Phys. Rev. ST Accel. Beams 17, 060702 (2014).

[16] T. Tanaka, X.-M. Maréchal, T. Hara, T. Tanabe, and H. Kitamura, J. Synchrotron Radiat. 5, 414 (1998).

[17] C. S. Hwang, J. C. Jan, C. S. Chang, S. D. Chen, C. H. Chang, and T. M. Uen, Phys. Rev. ST Accel. Beams 14, 044801 (2011).

[18] S. Sasaki, Nucl. Instrum. Methods Phys. Res., Sect. A 347, 83 (1994).

[19] C. W. Ostenfeld, F. Bødker, M.N. Pedersen, E. B. Christensen, M. B $\emptyset$ ttcher, and H. Bach, in Proceedings of the 22nd Particle Accelerator Conference, PAC-2007, Albuquerque, NM (IEEE, New York, 2007), p. TUPMN006.

[20] K. P. Wootton, M. J. Boland, B. C. C. Cowie, and R. P. Rassool, in Proceedings of the International Beams Instrumentation Conference 2013 (JACoW, Oxford, United Kingdom, 2013), p. TUPF19.

[21] T. Tanaka and H. Kitamura, J. Synchrotron Radiat. 8, 1221 (2001). 
[22] W. J. Corbett, M. J. Lee, and V. Ziemann, in Proceedings of the 15th Particle Accelerator Conference, PAC-1993, Washington, DC, 1993 (IEEE, New York, 1993), p. 108.

[23] J. Safranek, Nucl. Instrum. Methods Phys. Res., Sect. A 388, 27 (1997).

[24] R. Dowd, M. Boland, G. LeBlanc, and Y.-R. E. Tan, Phys. Rev. ST Accel. Beams 14, 012804 (2011).

[25] B. C. C. Cowie, A. Tadich, and L. Thomsen, AIP Conf. Proc. 1234, 307 (2010).

[26] J. Bahrdt, K. Holldack, P. Kuske, R. Müller, M. Scheer, and P. Schmid, Phys. Rev. Lett. 111, 034801 (2013).

[27] K. P. Wootton, G. N. Taylor, R. P. Rassool, M. J. Boland, B. C. C. Cowie, R. Dowd, Y.-R.E. Tan, and Y. Papaphilippou, in Proceedings of the International Beams Instrumentation Conference 2012 (JACoW, Tsukuba, Japan, 2012), p. MOCB04.

[28] A. Terebilo, in Proceedings of the Particle Accelerator Conference, Chicago, IL, 2001 (IEEE, New York, 2001), p. RPAH314.

[29] J. Corbett, G. Portmann, and A. Terebilo, in Proceedings of the 20th Particle Accelerator Conference, PAC2003, Portland, OR, 2003 (IEEE, New York, 2003), p. WPPE020.

[30] K. P. Wootton, M. J. Boland, B. C. C. Cowie, R. Dowd, and R. P. Rassool, in Proceedings of the International Beams Instrumentation Conference 2013 (JACoW, Oxford, United Kingdom, 2013), p. TUPF18.

[31] M. Sands, Report No. SLAC-R-121, 1970.

[32] T. O. Raubenheimer, Part. Accel. 36, 75 (1991).

[33] J. W. Boldeman and D. Einfeld, Nucl. Instrum. Methods Phys. Res., Sect. A 521, 306 (2004).
[34] A. W. Chao, J. Appl. Phys. 50, 595 (1979).

[35] T. Mitsuhashi, in Proceedings of the International Beams Instrumentation Conference 2012 (JACoW, Tsukuba, Japan, 2012), p. WEIC02.

[36] Å. Andersson, M. Böge, A. Lüdeke, V. Schlott, and A. Streun, Nucl. Instrum. Methods Phys. Res., Sect. A 591, 437 (2008).

[37] T. Moreno, E. Otero, and P. Ohresser, J. Synchrotron Radiat. 19, 179 (2012).

[38] Y. Cai, K. Bane, R. Hettel, Y. Nosochkov, M.-H. Wang, and M. Borland, Phys. Rev. ST Accel. Beams 15, 054002 (2012).

[39] G. Xu and Y. Jiao, Chin. Phys. C 37, 057003 (2013).

[40] L. Farvacque, N. Carmignani, J. Chavanne, A. Franchi, G. Le Bec, S. Liuzzo, B. Nash, T. Perron, and P. Raimondi, in Proceedings of the 4th International Particle Accelerator Conference, IPAC-2013, Shanghai, China, 2013 (JACoW, Shanghai, China, 2013), p. MOPEA008.

[41] M. Borland, G. Decker, L. Emery, V. Sajaev, Y. Sun, and A. Xiao, J. Synchrotron Radiat. 21, 912 (2014).

[42] R. Hettel, J. Synchrotron Radiat. 21, 843 (2014).

[43] N. O. Strelnikov and I. B. Vasserman, Phys. Rev. ST Accel. Beams 17, 062401 (2014).

[44] M. Eriksson, J. F. van der Veen, and C. Quitmann, J. Synchrotron Radiat. 21, 837 (2014).

[45] Y. Cai, Y. Ding, R. Hettel, Z. Huang, L. Wang, and L. Xiao, Synchrotron Radiat. News 26, 39 (2013).

[46] Y. Ding, P. Baxevanis, Y. Cai, Z. Huang, and R. D. Ruth, in Proceedings of the 4th International Particle Accelerator Conference, IPAC-2013, Shanghai, China, 2013 (JACoW, Shanghai, China, 2013), p. WEPWA075. 